\title{
EL INTERCAMBIO DE PRODUCTOS FORESTALES DIFERENTES DE LA MADERA EN EL AMBITO DE IQUITOS - PERU
}

\section{Juan Baluarte Vásquez ${ }^{1}$ y Mauro Vásquez Ramírez²}

\section{RESUMEN}

En el presente documento se reporta el volumen de venta mensual de los productos forestales no maderables, comercializados con mayor frecuencia en el mercado de Iquitos, y la participación de los principales centros de expendio en esta actividad económica. El 63\% de los productos forestales diferentes de la madera que se comercializan en Iquitos, corresponden a alimentos (frutas y hojas tiernas), el 15\% tienen uso medicinal, el $11 \%$ para artesanías y el $11 \%$ restante tiene otros usos. En el mercado de Morona Cocha se comercializa el 21\% del volumen de ventas, seguido de Belén y Bellavista-Nanay con 19 y $15 \%$, respectivamente. El volumen de comercialización mensual es de 184,41 t, correspondiendo a irapay (Lepidocaryum tenue) el $42 \%$. Cuatro productos: irapay (L. tenue), aguaje (Mauritia flexuosa), ungurahui (Oenocarpus bataua) y aguajillo (Mauritiella aculeata), congregan juntos el $84 \%$ del volumen de venta mensual. El importe mensual generado por la venta de los 27 productos comercializados es de S/. 63 716, correspondiendo a aguaje ( $M$. flexuosa), huambé (Philodendron sp.), irapay (L. tenue) y ungurahui (Oenocarpus bataua) el $56 \%$ de este monto; cada uno de los cuatro productos antes mencionados genera ingresos mensuales superiores a 5000 nuevos soles, constituyéndose en productos atractivos para la comercialización y sustento de los pobladores de la región.

Palabras clave: Comercialización, productos forestales diferentes de la madera, Amazonía Peruana.

1 Instituto de Investigaciones de la Amazonía Peruana. Avda. A. Quiñones km 2,5. E-mail: pet@iiap.org.pe; jbaluarte@iiap.org.pe

2 Proyecto Especial de Desarrollo Colombo-Peruano. Non Timber Forest Products Interchange around Iquitos-Peru. Calle Brasil 355. Iquitos (Perú). 


\begin{abstract}
Monthly volume sales of non timber forest products frequently commercialized in the Iquitos market and the participation of the main trading centers are reported in this paper. $63 \%$ of non timber forest products commercialized in Iquitos are connected to food (fruits and young leaves), $15 \%$ are used for medicine, $11 \%$ for handicraft, and the remaining $11 \%$ is derived to different uses. At Morona Cocha market $21 \%$ of the sales volume is commercialized, while in Belen and Bellavista-Nanay markets only the $19 \%$ and $15 \%$ are exchanged, respectively. Monthly commercialized volume is $184,41 \mathrm{t}$, where $42 \%$ corresponds to irapay (Lepidocaryum tenue). Four non timber forest products, irapay (L. tenue), aguaje (Mauritia flexuosa), ungurahui (Oenocarpus bataua) and aguajillo (Mauritiella aculeata) make altogether $84 \%$ of the monthly sales volume. Incomes generated for selling the 27 products is S/. 63 716, where aguaje ( $M$, flexuosa), haumbé (Philodendron sp.), irapay (L. tenue) and ungurahui (O. bataua) hold the $56 \%$ of this amount. Each product above mentioned provides monthly incomes higher than S/. 5 000, turning attractive this products both for interchanging and local people subsistence.
\end{abstract}

Key words: Commercialization, non timber forest products, Peruvian Amazon.

\title{
1. INTRODUCCION
}

A pesar de que la agricultura, principalmente de cultivos anuales, ha reemplazado a la extracción forestal como la actividad económica más importante en el ámbito de Iquitos, los productos forestales diferentes de la madera siguen contribuyendo en gran medida a la economía local (Vásquez y Gentry, 1989; Mejía, 1995; Padoch, 1990).

Debido a la enorme trascendencia que ha cobrado la comercialización de los productos del bosque, muchas familias de la región obtienen $50 \%$ o más de su ingreso monetario de estos productos (Padoch, 1990). Sin embargo, la comercialización de estos productos ha sido escasamente tratada en nuestra región, a excepción de los productos elaborados usando palmeras (Mejía, 1992; Pacheco, Espíritu e Hidalgo, 1992).

Los productos forestales diferentes de la madera representan uno de los grupos de productos más complejos desde el punto de vista de la comercialización debido a su número, versatilidad y variedad de aplicaciones (Lintu, 1995). 
Históricamente, la comercialización de los productos forestales diferentes de la madera, en la zona, está caracterizada por la aparición de auges pasajeros de ciertos productos, cuyas ganancias han sido escasamente revertidas en nuestra región.

Durante el período de ejecución del presente estudio -setiembre, octubre y noviembre de 1997-, correspondiente a la época de vaciante y media vaciante de los ríos de la región, se ha registrado y monitoreado la comercialización de 27 especies forestales diferentes de la madera de origen vegetal; de ellas, el $63 \%$ pertenece al rubro de alimentos (frutas y hojas tiernas), el $15 \%$ a medicinas, el $11,11 \%$ a artesanías, el $7,4 \%$ a materiales de construcción y el 3,7\% a hojas para empaquetar alimentos.

El escaso número de productos comercializados está, probablemente, relacionado con el período de ejecución del presente estudio ya que, como es conocido, gran parte de los productos que se comercializan en Iquitos, principalmente frutas, tienen carácter estacional.

\section{MATERIALES Y METODOS}

\subsection{Jurisdicción y características de la zona donde se realizó el trabajo}

El presente estudio se realizó en el ámbito de las ciudades de Iquitos y Punchana. Ambas ciudades pertenecen a la provincia de Maynas, región Loreto, $03^{\circ} 45^{\prime} \mathrm{S}$ y $73^{\circ} 15^{\prime} \mathrm{O}$, a una altitud de $126 \mathrm{msnm}$. Presentan un clima con las siguientes características: precipitación media anual, $2876,5 \mathrm{~mm}$; temperatura media, $24,3^{\circ} \mathrm{C}$ y humedad relativa promedio mensual, $80 \%$.

\subsection{Características de los centros de expendio monitoreados durante el presente trabajo}

Los centros de expendio seleccionados para el presente trabajo se indican en el Cuadro 1 . Allí, se resaltan las características más importantes como ubicación y accesibilidad. 
Cuadro 1. Principales centros de expendio de productos forestales diferentes de la madera en el ámbito de Iquitos.

\begin{tabular}{|c|c|}
\hline CENTROS DE EXPENDIO & CARACTERISTICAS \\
\hline ASTORIA & $\begin{array}{l}\text { Ubicado al norte de la ciudad de Iquitos, distrito } \\
\text { de Punchana, a orillas del río Amazonas, en la } \\
\text { Av. de la Marina, fácil acceso. En años anterio- } \\
\text { res uno de los centros de mayor acopio de pro- } \\
\text { ductos forestales diferentes de la madera que eran } \\
\text { exportados a países europeos, principalmente. }\end{array}$ \\
\hline BELLAVISTA - NANAY & $\begin{array}{l}\text { Ubicado al norte de la ciudad de Iquitos, en el } \\
\text { distrito de Punchana, a orillas del río Nanay. La } \\
\text { accesibilidad es fácil ya que el lugar es paradero } \\
\text { de muchas líneas de transporte. }\end{array}$ \\
\hline $\begin{array}{l}\text { MERCADILLO MORONA } \\
\text { COCHA Y ALREDEDORES }\end{array}$ & $\begin{array}{l}\text { En esta zona se encuentra el mayor número de } \\
\text { centros de acopio y comercialización de hojas } \\
\text { para cubiertas de techo, principalmente de irapay, } \\
\text { yarina y otros. Está ubicado al lado oeste de la } \\
\text { ciudad, frente al lago de Morona Cocha, en la } \\
\text { Av. del Ejército, curva de Morona Cocha. Es de } \\
\text { fácil acceso. }\end{array}$ \\
\hline P. BELEN Y ALREDEDORES & $\begin{array}{l}\text { Ubicado en la parte sur-este de la ciudad, en la } \\
\text { desembocadura del río Itaya al río Amazonas. } \\
\text { Es el centro de acopio y comercialización más } \\
\text { grande de Iquitos. }\end{array}$ \\
\hline MERCADILLO "LA NORTEÑITA" & $\begin{array}{l}\text { Ubicado en la parte nor-oeste de la ciudad de } \\
\text { Iquitos en las intersecciones de las calles } \\
\text { Putumayo y Manco Capac. De fácil acceso. Se } \\
\text { caracteriza por no ser un centro de acopio direc- } \\
\text { to, ya que los productos forestales diferentes de } \\
\text { la madera que se ofertan son adquiridos en cen- } \\
\text { tros de acopio mayores. }\end{array}$ \\
\hline MERCADO DE PRODUCTORES & $\begin{array}{l}\text { Ubicado en la parte nor-este de la ciudad, a ori- } \\
\text { llas del río Amazonas, en la intersección de las } \\
\text { calles La Condamine con Requena. Cuenta con } \\
\text { infraestructura nueva para el acopio y } \\
\text { comercialización de productos. De fácil acceso. }\end{array}$ \\
\hline
\end{tabular}


Continúa...

\begin{tabular}{|l|l|}
\hline \multicolumn{1}{|c|}{ CENTROS DE EXPENDIO } & \multicolumn{1}{c|}{ CARACTERISTICAS } \\
\hline QUISTOCOCHA & $\begin{array}{l}\text { Ubicado a } 42 \text { minutos aproximadamente de la } \\
\text { ciudad, con un recorrido de } 13 \mathrm{~km} \text { por la carrete- } \\
\text { ra Iquitos-Nauta; el acceso es a través de la vía } \\
\text { terrestre. En dicho caserío se encuentra el Par- } \\
\text { que Zoológico de Quistococha. }\end{array}$ \\
\hline RUMOCOCHA & $\begin{array}{l}\text { Ubicado a } 20 \text { minutos aproximadamente de la } \\
\text { ciudad, con un recorrido de } 6 \text { km por la carretera } \\
\text { que conduce a Santa Clara. El acceso es por vía } \\
\text { terrestre y fluvial a través del río Nanay. Está a } \\
\text { orillas del río Nanay. }\end{array}$ \\
\hline SANTA CLARA & $\begin{array}{l}\text { Uno de los balnearios más importantes de Iquitos, } \\
\text { ubicado 48 minutos aproximadamente de la ciu- } \\
\text { dad, con un recorrido de } 15 \mathrm{~km} \text {. El acceso es por } \\
\text { vía terrestre y fluvial por el río Nanay. Está a } \\
\text { orillas del río Nanay. }\end{array}$ \\
\hline SANTO TOMAS & $\begin{array}{l}\text { Balneario turístico, ubicado a } 50 \text { minutos aproxi- } \\
\text { madamente, con un recorrido de } 16 \text { km. El acce- } \\
\text { so es por vía terrestre y fluvial a través del río } \\
\text { Nanay. }\end{array}$ \\
\hline
\end{tabular}

Fuente: Recolección de información en trabajos de campo.

El procedimiento consistió en visitas de una vez por semana durante tres meses (setiembre-noviembre 1997) a los centros de expendio seleccionados para el presente trabajo. Este seguimiento permitió conocer el volumen de comercialización diario y mensual, el precio unitario de cada producto y el monto total recaudado diaria y mensualmente por la venta de los productos forestales diferentes de la madera.

\subsection{Especies de productos forestales diferentes de la madera}

El monitoreo de la comercialización de los productos forestales diferentes de la madera, se ha limitado al registro de 27 especies de origen vegetal, que se comercializan con mayor frecuencia en los centros de expendio arriba citados. 


\section{RESULTADOS Y DISCUSIONES}

\subsection{Centros de expendio monitoreados durante el presente trabajo}

Los centros de comercialización monitoreados en el presente estudio han sido identificados en base a la accesibilidad de los extractores e intermediarios para la comercialización de los productos.

Los centros de expendio ubicados en poblados cercanos a Iquitos y en la ribera del río Nanay, están caracterizados por la venta directa de los extractores de estos productos; entre estos lugares están Bellavista-Nanay, Morona Cocha y alrededores, Santa Clara, Rumococha y Santo Tomás.

El mercadillo de Morona Cocha y alrededores se ha especializado en la venta de irapay, por esta razón este lugar aporta el $37 \%$ de la venta mensual de este producto en Iquitos, cuyo monto de comercialización mensual es similar a los resultados reportados por Pacheco, Espíritu e Hidalgo (1992). En general, este mercadillo es el mayor centro de comercialización de productos diferentes de la madera, allí se comercializa el $21 \%$ del volumen de ventas mensuales de productos forestales diferentes de la madera, seguido de los mercados de Belén y Bellavista-Nanay con 19\% y $15 \%$, respectivamente. En estos tres mercados juntos se comercializa el 55\% de los productos forestales diferentes de la madera, en Iquitos. Esta realidad nos permite sugerir que el control de estos productos se centraliza en estos tres principales mercados.

No obstante, constituir el mercado de Morona Cocha en el principal centro de comercialización de productos forestales diferentes de la madera, contribuye con una menor variedad de productos que se comercializan en el ámbito de Iquitos. En este aspecto, el mercado de Belén muestra una mayor variedad (89\%), seguido del mercado Bellavista-Nanay con $85 \%$ de los productos que se comercializan en el ámbito de Iquitos.

La comercialización de estos productos en los centros de expendio citados, se desarrolla libremente, utilizando diversas unidades de medida, dependiendo del tipo y cantidad del producto, pudiendo ser sacos, rollos o porciones.

La existencia de este mercado paralelo, escasamente controlado por el sector agrario, demuestra que esta actividad se desarrolla dentro de un contexto bastante informal, 
perjudicando al Estado peruano por la evasión de impuestos que generaría el aprovechamiento de este recurso.

\subsection{Especies de productos forestales diferentes de la madera que se comercializan en el ámbito de Iquitos}

Las 27 especies que proporcionan productos forestales diferentes de la madera, que se comercializan en los centros de expendio del ámbito de Iquitos, se clasifican en: (a) alimenticios (frutas y hojas tiernas), 63\%; (b) medicinales, 15\%; (c) artesanales, 11\%; (d) materiales de construcción, 7\%; (e) empaques de alimentos, 4\% (Figura 1).

Esta situación refleja que la población amazónica utiliza los productos del bosque para satisfacer sus necesidades elementales como alimentación, salud y vivienda; siendo estos productos de trascendental importancia para la vida del poblador de esta región del país.

El número de especies, evaluadas en este informe, que proporcionan productos forestales diferentes de la madera, es inferior a los resultados reportados por Vásquez y Gentry (1989); Padoch (1990) y Mejía (1995). Este escaso número de especies está relacionado con el período en el que se desarrolló el presente estudio, ya que muchos de los productos forestales diferentes de la madera, son estacionales, especialmente los frutos.

\subsection{Volumen comercializado e ingresos generados en los centros de venta monitoreados}

En el Cuadro 2 del Anexo, se presentan los montos comercializados en toneladas ( $\mathrm{t}$ ) y nuevos soles (S/.) por trimestre y mensual, generado por la comercialización de productos forestales diferentes de la madera, en los principales centros de expendio del ámbito de Iquitos. El Cuadro 3 resume esta información; allí, se puede apreciar que el volumen de venta mensual de estos productos asciende a 184401 toneladas equivalente a S/. 63716 . 
Cuadro 2. Comercialización de productos forestales diferentes de la madera en los principales centros de expendio del ámbito de Iquitos, monitoreados durante tres meses (setiembre a noviembre de 1997).

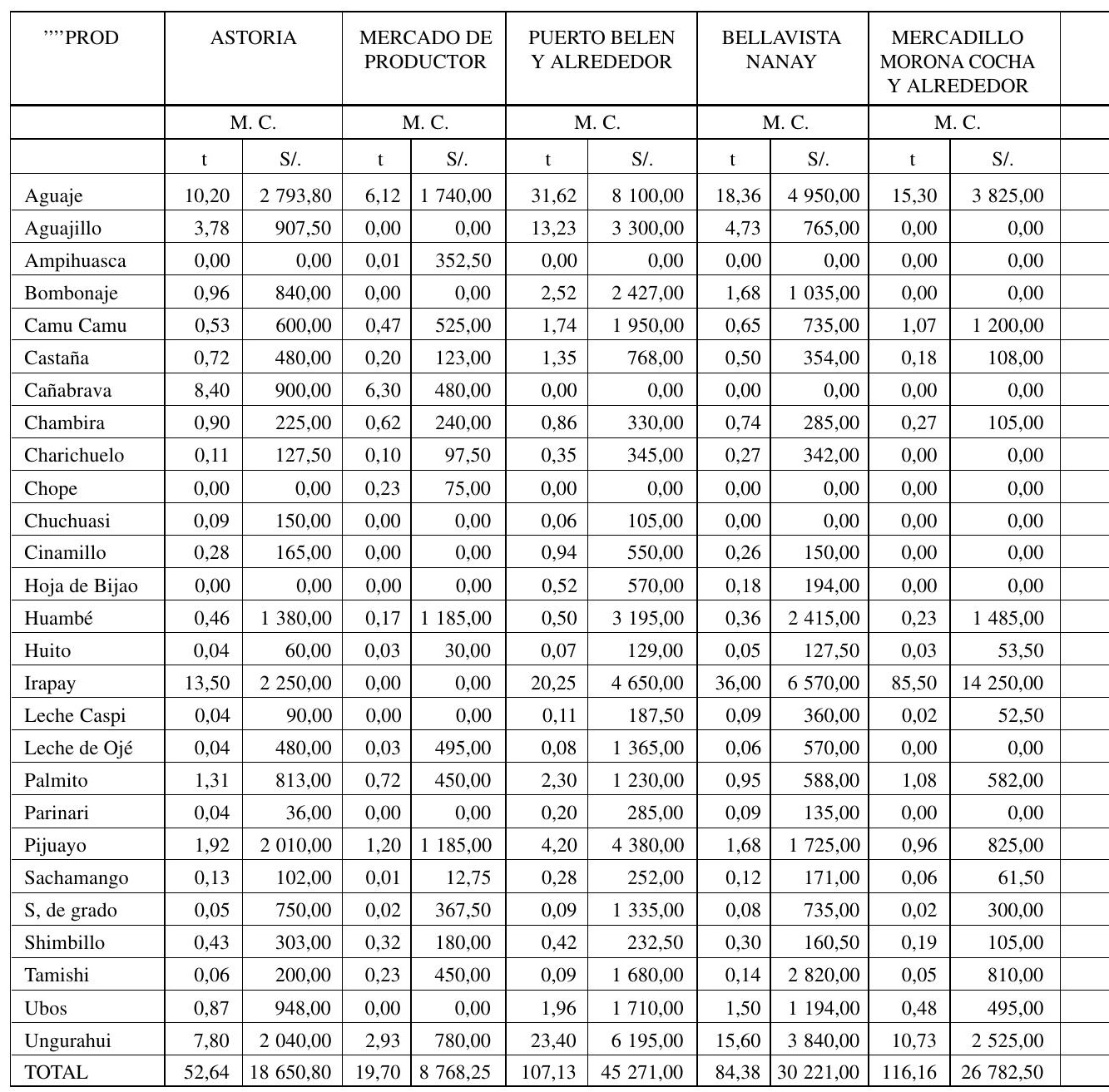

M.C. $=$ Monto de comercialización $\mathrm{t}=$ toneladas 


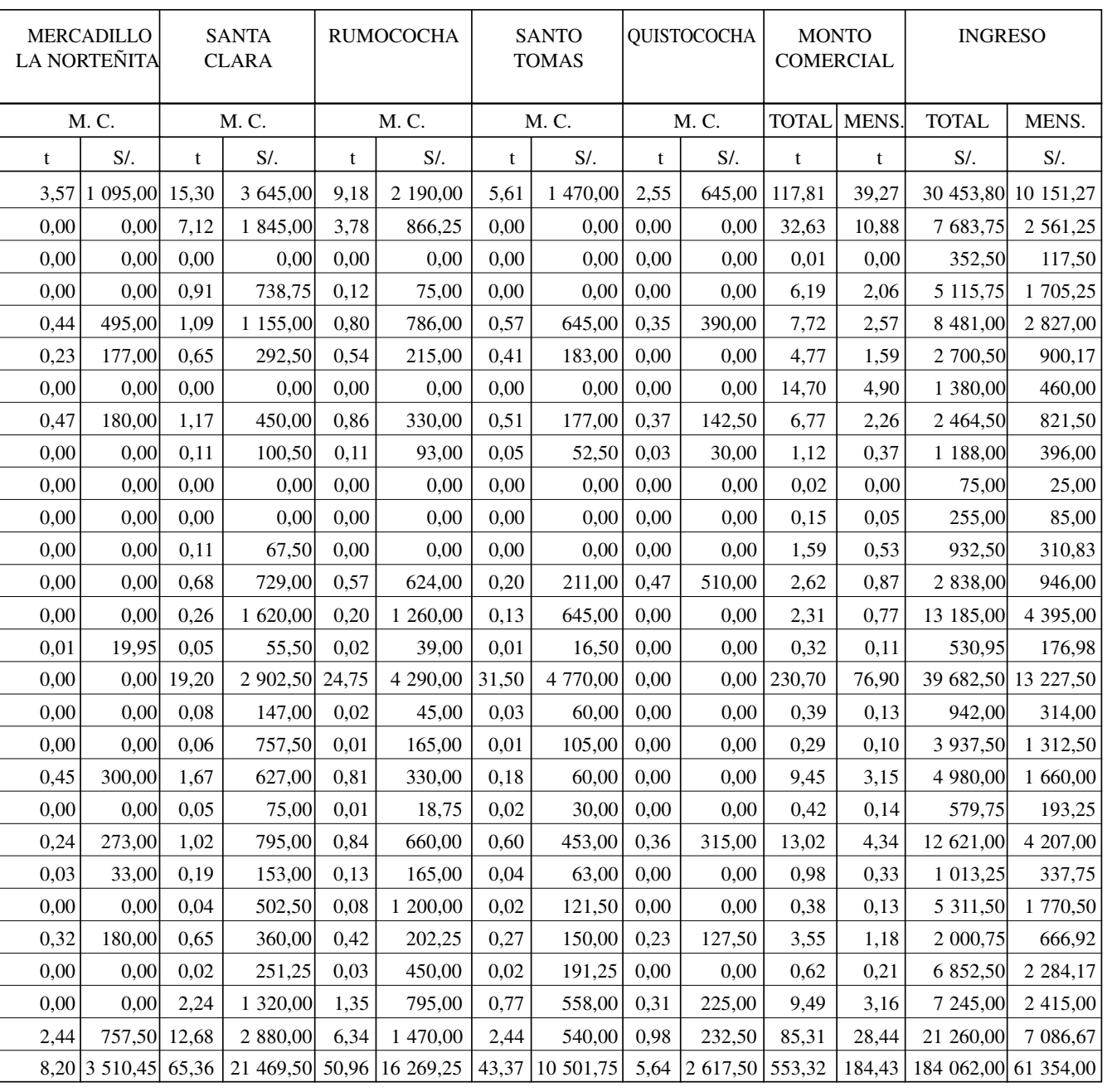


Cuadro 3. Volumen e ingreso mensual de productos diferentes de la madera comercializados en los centros monitoreados.

\begin{tabular}{|c|c|c|c|c|c|c|}
\hline \multirow[t]{2}{*}{$\mathbf{N}^{\circ}$ PRODUCTO } & \multirow{2}{*}{$\begin{array}{c}\text { UNIDAD } \\
\text { DE } \\
\text { MEDIDA } \\
\text { UM } \\
\end{array}$} & \multirow{2}{*}{$\begin{array}{c}\text { EQUIVAL. } \\
\text { PROM/UM } \\
\quad(\mathrm{Kg})\end{array}$} & \multirow{2}{*}{$\begin{array}{c}\text { PRECIO } \\
\text { UNIT./UM } \\
\text { (S/.) }\end{array}$} & \multicolumn{3}{|c|}{ VENTA MENSUAL } \\
\hline & & & & $\mathrm{UM}$ & $(\mathrm{t})$ & $(\mathrm{S} /)$. \\
\hline 1. Aguaje & Saca & 68,00 & 17,56 & 577,00 & 39,270 & 10152 \\
\hline 2. Aguajillo & Saca & 63,00 & 14,85 & 172,50 & 10,867 & 2561 \\
\hline 3. Ampihuasca & Rollo & 0,90 & 23,50 & 5,00 & 0,004 & 117 \\
\hline 4. Bijao & Rollo & 0,25 & 0,27 & 3480,00 & 0,870 & 939 \\
\hline 5. Bombonaje & Carga & 8,00 & 6,62 & 257,50 & 2,060 & 1704 \\
\hline 6. Camu-camu & Bolsa & 0,44 & 0,49 & 5780,00 & 2,572 & 2832 \\
\hline 7. Cañabrava & Ciento & 140,00 & 13,14 & 35,00 & 4,900 & 460 \\
\hline 8. Castaña & Fruto & 1,50 & 0,85 & 1060,00 & 1,590 & 901 \\
\hline 9. Cinamillo & Porción & 0,85 & 0,50 & 625,00 & 0,531 & 312 \\
\hline 10. Chambira & Porción & 1,30 & 0,47 & 1735,00 & 2,255 & 815 \\
\hline 11. Charichuelo & Porción & 0,50 & 0,53 & 746,00 & 0,373 & 395 \\
\hline 12. Chopé & Porción & 0,30 & 1,00 & 25,00 & 0,007 & 25 \\
\hline 13. Chuchuhuasi & Porción & 0,60 & 1,00 & 85,00 & 0,051 & 85 \\
\hline 14. Huambé & Rollo & 2,20 & 14,33 & 350,00 & 0,770 & 5015 \\
\hline 15. Huasaí & Unidad & 1,50 & 0,79 & 2100,00 & 3,150 & 1659 \\
\hline 16. Huito & Unidad & 0,10 & 0,17 & 1052,00 & 0,105 & 179 \\
\hline 17. Irapay & Ciento & 150,00 & 25,85 & 512,50 & 76,875 & 13248 \\
\hline 18. Leche huayo & Bolsa & 0,25 & 0,57 & 550,00 & 0,137 & 313 \\
\hline 19. Ojé & Botella & 0,50 & 6,91 & 190,00 & 0,095 & 1313 \\
\hline 20. Parinari & Porción & 0,35 & 0,48 & 402,50 & 0,140 & 193 \\
\hline 21. Pijuayo & Racimo & 4,00 & 3,88 & 1085,00 & 4,340 & 4210 \\
\hline 22. Sachamango & Unidad & 0,25 & 0,26 & 1310,00 & 0,327 & 340 \\
\hline 23. Sangre de grado & Botella & 0,50 & 7,01 & 252,50 & 0,126 & 1170 \\
\hline 24. Shimbillo & Porción & 0,90 & 0,51 & 1315,00 & 1,183 & 670 \\
\hline 25. Tamshi & Rollo & 0,50 & 9,52 & 420,00 & 0,210 & 3998 \\
\hline 26. Ubos & Bolsa & 0,85 & 0,65 & 3720,00 & 3,162 & 2418 \\
\hline 27. Ungurahui & Saca & 65,00 & 16,21 & 437,50 & 28,437 & 7097 \\
\hline TOTAL & & -- & -- & - & 184,401 & 63716 \\
\hline
\end{tabular}

Las Figuras 2 y 3 muestran los productos con mayor volumen de venta e ingresos monetarios mensuales generados por la comercialización en los mercados del ámbito de Iquitos.

Cuatro productos (irapay, aguaje, ungurahui y aguajillo) generan juntos el $84 \%$ del volumen de venta mensual (Figura 2). 
Asimismo, cuatro productos (aguaje, huambé, irapay y ungurahui) congregan juntos el 56\% de los ingresos monetarios generados por la venta de estos productos (Figura 3 ). Se observa que irapay es el producto que genera mayor ingreso $(21 \%)$, seguido de aguaje, ungurahui y huambé con 16,11 y $8 \%$, respectivamente. Cada uno de estos cuatro productos genera ingresos que superan los 5000 nuevos soles mensuales.

Esta realidad nos permite afirmar que algunos productos forestales diferentes de la madera, constituyen un atractivo negocio para muchos pobladores de Iquitos.

\section{BIBLIOGRAFIA}

LINTU, L. 1995. La comercialización de los productos forestales no maderables en los países en desarrollo. En: Unasylva 46 (183): 37 - 41.

MEJIA, K. 1992. Las palmeras en los mercados de Iquitos. En: Bull. de l'Institut Francais d'Etudes Andines 21(2): 755-769.

1995. Diagnóstico de los recursos vegetales de la Amazonía Peruana. Documento Técnico No 16. Iquitos, Perú: IIAP. 59 pp.

PACHECO, T.; ESPIRITU, J.; HIDALGO, J. 1992. Comercialización de hojas de palmeras utilizadas como techos en Iquitos, Perú. En: Conocimiento 2 (2-3). pp. 165-174.

PADOCH, C. 1990. Importancia económica y comercialización de los productos del bosque y de las purmas en la región de Iquitos. En: Denevan; Padoch (editores). Agroforestería tradicional en la Amazonía Peruana. Documento 11. Lima: CIPA. pp.162-193.

VASQUEZ, R.; A.H. GENTRY. 1989. Use and misuse of forest harvested fruits in the Iquitos area. In: Conservation Biology, 3(4): 1-11. 


\section{Figura 1}

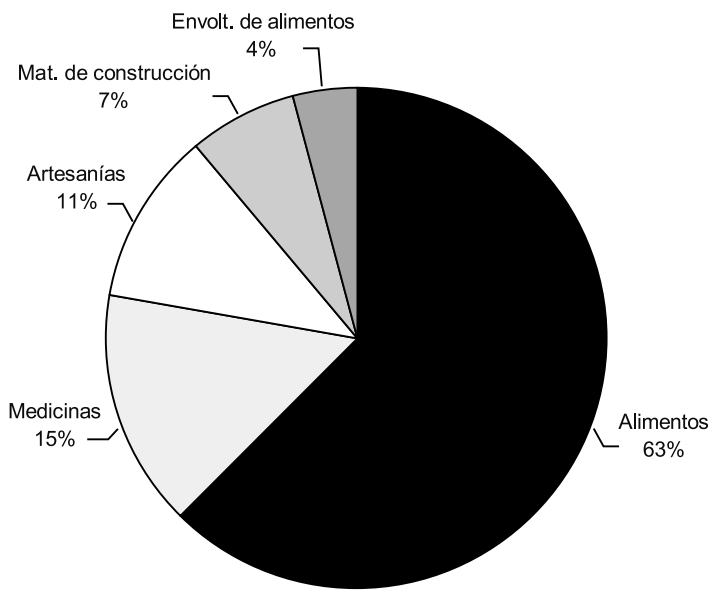

Fig. 1. Clasificación de productos forestales diferentes de la madera comercializados en el ámbito de Iquitos.

Figura 2

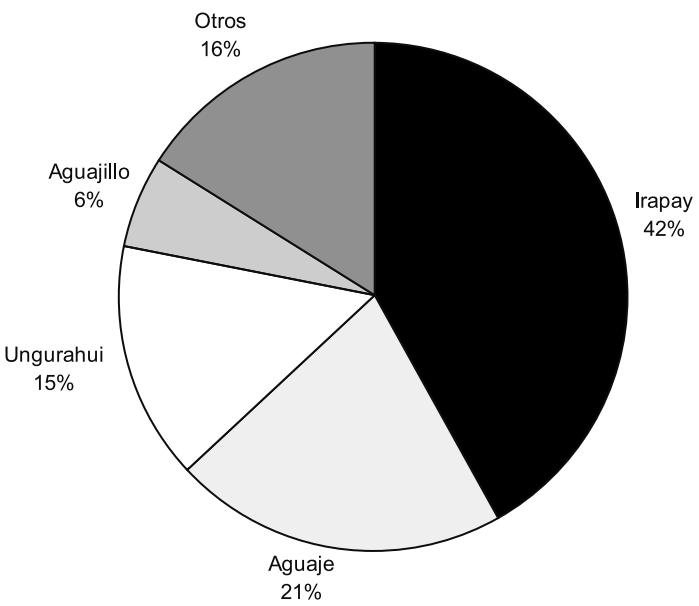

Fig. 2 Productos con mayor volumen de venta mensual. 


\section{Figura 3}

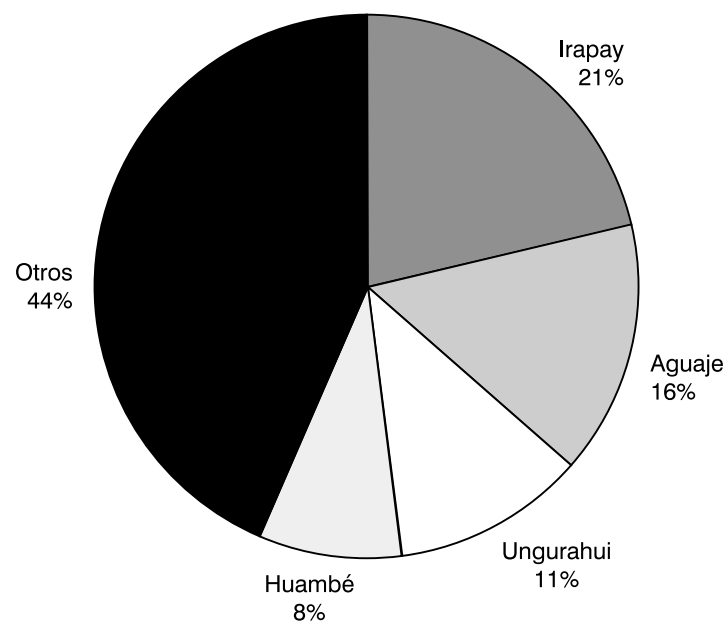

Fig. 3. Productos que generan mayores ingresos monetarios mensuales. 